\title{
EFEITO DO MÉTODO DE COMPLEXAÇÃO COMBINANDO EDTA - CITRATO E COPRECIPITAÇÃO EM MEIO OXALATO NA SÍNTESE DA $\mathrm{SrCo}_{0,8} \mathrm{Fe}_{0,2} \mathrm{O}_{3-\delta}$
}

\author{
A. G. SANTOS ${ }^{1 *}$, I. K. F. $\operatorname{COSTA}^{1}$, F. K. G. SANTOS ${ }^{1}$ e C. P. SOUZA ${ }^{2}$ \\ ${ }^{1}$ Departamento de Agrotecnologia e Ciências Sociais - Universidade Federal Rural do Semi-Árido \\ ${ }^{2}$ Departamento de Engenharia Química - Universidade Federal do Rio Grande do Norte \\ andarair@ufersa.edu.br
}

Artigo submetido em janeiro/2014 e aceito em maio/2015

DOI: $10.15628 /$ holos.2015.2160

\section{RESUMO}

Este trabalho enfoca o método de síntese de coprecipitação para preparação da perovsquita $\mathrm{SrCO}_{0,8} \mathrm{Fe}_{0,2} \mathrm{O}_{3-\delta}$ e suas caracterizações, desenvolvendo uma rota metodológica alternativa de síntese a partir da coprecipitação via meio oxalato. No intuito de verificar a influência do método, a perovsquita foi sintetizada também com base no método de complexação combinando EDTA - Citrato. Ambos os métodos de síntese permitiram obter pós monofásicos. A síntese com base no método via coprecipitação em meio oxalato permitiu obter tamanho de partículas na mesma ordem de grandeza que os obtidos a partir do método de complexação combinando EDTA - Citrato. A natureza do reagente utilizado no método via coprecipitação em meio oxalato produziu um material com aproximadamente $80 \%$ menor em relação ao tamanho médio de cristalito. Além disso, o método via coprecipitação em meio oxalato obteve precursores em estado sólido, em baixa temperatura $\left(26^{\circ} \mathrm{C}\right)$, menor tempo de síntese, maior estabilidade térmica e um rendimento maior da ordem 90 a $95 \%$, mantendo na mesma ordem de grandeza o tamanho médio de cristalito que o método de complexação combinando EDTA - Citrato.

PALAVRAS-CHAVE: perovsquita, síntese via coprecipitação, oxalato, método EDTA-citrato, método Rietveld.

\section{EFFECT OF COMBINED CITRATE - EDTA COMPLEXING METHOD AND COPRECIPITATION OXALATE THE SYNTHESIS OF $\mathrm{SrCO}_{0.8} \mathrm{Fe}_{0.2} \mathrm{O}_{3-\delta}$}

\begin{abstract}
This work focuses on the coprecipitation synthesis method for preparation of ceramic materials with perovskite structure and characterization, developing a methodological alternative route of synthesis from the middle via oxalate coprecipitation material $\mathrm{SrCO}_{0.8} \mathrm{Fe}_{0.2} \mathrm{O}_{3-\delta}$. In order to check the influence of this method, it was also synthesized using a combined citrate - EDTA complexing method. Both methods led to post-phase synthesis. The synthesis based on the method via oxalate coprecipitation among particles led to the crystalline phase as those obtained using a
\end{abstract}

combined citrate - EDTA complexing method under the same conditions of heat treatment. The nature of the reagent used via oxalate coprecipitation method produced a material with approximately $80 \%$ lower than the average size of crystallites. Moreover, the via oxalate coprecipitation method precursors obtained in the solid state at low temperature $\left(26^{\circ} \mathrm{C}\right)$, shorter synthesis, greater thermal stability and a higher yield of around 90-95 \%, maintaining the same order of magnitude the crystallite size that the combined citrate EDTA complexing method.

KEYWORDS: perovskite, co-precipitation, oxalate, combined citrate - EDTA complexing, Rietveld method. 


\section{INTRODUÇÃO}

Nas últimas décadas, as membranas cerâmicas inorgânicas têm recebido maiores atenções em pesquisas e desenvolvimento, devido apresentarem alta condutividade mista (iônica e elétrica) e permeabilidade de oxigênio (Teraoka et al., 1985; Teraoka et al., 1988; Shao et al., 2001; Mineshige et al., 2005; Mcintosh et al., 2006), sua mais importante propriedade uma vez que está diretamente relacionada à sua aplicação, podendo operar a temperaturas elevadas e em ambientes corrosivos, típicos de muitos processos industriais (Rangel, 1997). Essas membranas cerâmicas são materiais promissores para muitos processos industriais que requerem um fornecimento continuo de oxigênio puro, como em processos Fischer-Trosphs e síntese de metanol. Todavia, quando esses materiais são submetidos a altas temperaturas podem ocorrer mudanças de fase e/ou estruturais. As mudanças estruturais podem também ser induzidas pelos repetidos ciclos de sorção e dessorção da substância que permeia, por exemplo, oxigênio. A partir daí, muitos estudos tem sido realizados para minimizar os problemas que ocorrem com esses materiais. Tendo, por sua vez, como o maior desafio à busca de um material, com condições de síntese e/ou operação reacional que associe as propriedades de alta permeabilidade de oxigênio e alta estabilidade termodinâmica em um único material.

Os óxidos simples ou mistos têm sido investigados também como catalisadores para produção de biodiesel, sendo comum o uso dos óxidos: CaO (Macleod et al., 2008; Nakatani et al., 2009), La- $\mathrm{ZrO}_{2}$ (Yan et al., 2009), (CuO (Su et al., 2013), $\mathrm{TiO}_{2}-\mathrm{ZnO}$ (Madhuvilakku et al., 2013), entre outros. Alguns trabalhos têm demonstrado a versatilidade das perovsquitas, tais como: na redução de $\mathrm{NO}$ com $\mathrm{CO}_{2}$ ou hidrogênio para produzir $\mathrm{CO}_{2}$ e $\mathrm{N}_{2}$ (Stathopoulos et al., 2009), na oxidação total de etanol (Soares et al., 2007), na conversão do metano a gás de síntese pela reforma com $\mathrm{CO}_{2}$ (Lima et al., 2007), entre outras; evidenciam a versatilidade dos óxidos.

As propriedades catalíticas da perovsquita $\mathrm{SrCO}_{0,8} \mathrm{Fe}_{0,2} \mathrm{O}_{3-\delta}$ foi testada também na reação de oxidação do $\mathrm{CO}$ a $\mathrm{CO}_{2}$ numa faixa de temperatura de $75-300{ }^{\circ} \mathrm{C}$ por Santos et al., (2014). Conforme resultados, apesar da pequena área superficial apresentada pelas perovsquitas, o monóxido de carbono foi completamente convertido, mantendo sua estabilidade e sendo facilmente regenerado em baixa temperatura $\left(400{ }^{\circ} \mathrm{C}\right)$. Ainda segundo os autores, $\mathrm{O}$ comportamento da atividade catalítica do material pode está correlacionada ao tamanho médio de cristalito apresentado pela perovsquita.

A mistura de óxidos de composição $\mathrm{SrCo}_{0,8} \mathrm{Fe}_{0,2} \mathrm{O}_{3-\delta}$ do tipo perovsquita sintetizada pelo método de complexação combinando EDTA - Citrato (Mcintosh et al., 2006) é bem rediscutida na literatura (Teraoka et al., 1985; Qiu et al., 1995; Liu et al., 1996; Lee et al., 1997; Zhang et al., 1999; Shao et al., 2001; Tong et al., 2002; Fan et al., 2004, Minishige et al., 2005; Mcintosh et al., 2006; Fan et al., 2007). Todavia, apesar do uso do mesmo método de síntese observam-se variações diversas nas metodologias, porém são poucas as informações encontradas da abordagem do método de síntese e principalmente da sua influência nas caracterizações dos pós. Segundo, lkeguche et al., (2005) o método de preparação dos materiais possivelmente influencia as características físico-químicas do material, como: a microestrutura e a cristalinidade, e na uniformidade da composição local. 
Neste âmbito, o presente trabalho tem como objetivo principal avaliar as propriedades cristalográficas e morfológicas da perovsquita $\mathrm{SrCO}_{0,8} \mathrm{Fe}_{0,2} \mathrm{O}_{3-\delta}$ quando sintetizada pelos métodos de coprecipitação em meio oxalato e complexação combinando EDTA - Citrato, verificando ainda a influência de modificações nas variáveis que compõem as metodologias testadas. Finalmente, será proposta uma rota de síntese com base nos métodos em questão, de forma que se apresente de maneira eficiente e com facilidade de processamento, reprodutibilidade e confiabilidade de produção dos pós.

\section{EXPERIMENTAL}

\subsection{Reagentes utilizados}

A síntese dos materiais cerâmicos de estrutura perovsquita de composição $\mathrm{SrCo}_{0,8} \mathrm{Fe}_{0,2} \mathrm{O}_{3-\delta}$ foi realizada com base no método de complexação combinando EDTA - Citrato e no método de coprecipitação em meio oxalato, os reagentes utilizados em ambos métodos estão resumidos na Tabela 1.

Tabela 1 - Lista de reagentes químicos utilizados nas sínteses $\left(\mathrm{SrCO}_{0,8} \mathrm{Fe}_{0,2} \mathrm{O}_{3-\delta}\right)$.

\begin{tabular}{|c|c|c|c|}
\hline REAGENTE & FÓRMULA QUÍMICA & PUREZA, \% & FABRICANTE \\
\hline \multicolumn{4}{|c|}{ Método de complexação combinando EDTA-Citrato } \\
\hline EDTA ácido & $\mathrm{C}_{10} \mathrm{H}_{16} \mathrm{~N}_{2} \mathrm{O}_{8}$ & 99,99 & Sigma/Aldrick \\
\hline Nitrato de estrôncio & $\mathrm{Sr}\left(\mathrm{NO}_{3}\right)_{2}$ & 99 & Sigma/Aldrick \\
\hline Nitrato de cobalto II hexahidratado & $\mathrm{Co}\left(\mathrm{NO}_{3}\right)_{2} 6 \mathrm{H}_{2} \mathrm{O}$ & 98 & Sigma/Aldrick \\
\hline Nitrato de ferro III nanohidratado & $\mathrm{Fe}\left(\mathrm{NO}_{3}\right)_{3} 9 \mathrm{H}_{2} \mathrm{O}$ & 98 & Sigma/Aldrick \\
\hline Hidróxido de amônia & $\mathrm{NH}_{4} \mathrm{OH}$ & 25 & Fluka \\
\hline Ácido cítrico & $\mathrm{C}_{6} \mathrm{H}_{8} \mathrm{O}_{7}$ & 99 & Sigma/Aldrick \\
\hline \multicolumn{4}{|c|}{ Método de coprecipitação em meio oxalato } \\
\hline Carbonato de estrôncio & $\left(\mathrm{SrCO}_{3}\right)$ & 99,99 & Sigma/Aldrick \\
\hline Nitrato de cobalto II hexahidratado & $\left(\mathrm{Co}\left(\mathrm{NO}_{3}\right)_{2} 6 \mathrm{H}_{2} \mathrm{O}\right)$ & 98 & Sigma/Aldrick \\
\hline Oxalato de ferro III hexahidratado & $\mathrm{Fe}\left(\mathrm{C}_{2} \mathrm{O}_{4}\right)_{3} 6 \mathrm{H}_{2} \mathrm{O}$ & 98 & Sigma/Aldrick \\
\hline Nitrato de ferro III nanohidratado & $\mathrm{Fe}\left(\mathrm{NO}_{3}\right)_{3} 9 \mathrm{H}_{2} \mathrm{O}$ & 98 & Sigma/Aldrick \\
\hline Trietilamina & $\mathrm{C}_{6} \mathrm{H}_{15} \mathrm{~N}$ & 99 & Sigma/Aldrick \\
\hline Ácido oxalato & $\mathrm{C}_{6} \mathrm{H}_{8} \mathrm{O}_{7}$ & 99 & Sigma/Aldrick \\
\hline Oxalato de amônia & $\left(\mathrm{NH}_{4}\right)_{2} \mathrm{C}_{2} \mathrm{O}_{4} \mathrm{H}_{2} \mathrm{O}$ & 99 & Sigma/Aldrick \\
\hline
\end{tabular}

\subsection{Metodologia de síntese}

\subsubsection{Método EDTA-Citrato}

Inicialmente, pós de composição $\mathrm{SrCO}_{0,8} \mathrm{Fe}_{0,2} \mathrm{O}_{3-\delta}$, foram investigados e reproduzidas metodologias abordadas na literatura (Shao et al., 2001; Tong et al., 2002; Ikeguche et al., 2005). Dentro deste contexto, foi proposta uma metodologia com a finalidade de dominar o método de síntese para preparação do pó e conhecer as características finais dos pós.

Para síntese dos pós, um aquecedor/agitador (modelo Fisaton) foi utilizado na etapa de obtenção do gel, juntamente com um termômetro e um pHmetro (modelo PHS-3B, PHTEK) para controle de temperatura e $\mathrm{pH}$, respectivamente. Para etapa de calcinação foi utilizado um forno tipo mufla (EDG) com temperatura e aquecimento programável. 
A metodologia de síntese proposta com base no método de complexação combinando EDTA - Citrato é descrita a seguir: quantidade apropriada de EDTA ácido foi diluída em hidróxido de amônia numa razão $1 \mathrm{~g}: 10 \mathrm{~mL}$ e mantida sob agitação e aquecimento (30 $-40{ }^{\circ} \mathrm{C}$ ) até obtenção de uma solução límpida (solução 1), em seguida a solução 2, à base dos elementos ( $\mathrm{Sr}$, Co e Fe) e em proporções definidas estequiometricamente foi adicionada a solução 1 , por fim, o ácido cítrico foi adicionado (solução 3) e a temperatura controlada em cerca de $80{ }^{\circ} \mathrm{C}$ e o pH até a formação do gel. Uma razão molar de EDTA ácido $\left(\mathrm{C}_{10} \mathrm{H}_{16} \mathrm{~N}_{2} \mathrm{O}_{8}\right)$, ácido cítrico $\left(\mathrm{C}_{6} \mathrm{H}_{8} \mathrm{O}_{7}\right)$ e íons metálicos totais de 1:1,5:1 foi adotada para obtenção do material cerâmico do tipo perovsquita. Em caso de precipitação, o pH da solução foi corrigido com adição de $\mathrm{NH}_{4} \mathrm{OH} 25$ \%. A Figura 1 mostra a rota metodológica proposta como procedimento experimental para síntese do material cerâmico $\left(\mathrm{SrCO}_{0,8} \mathrm{Fe}_{0,2} \mathrm{O}_{3-\delta}\right)$ com base no método de complexação combinando EDTA - Citrato.

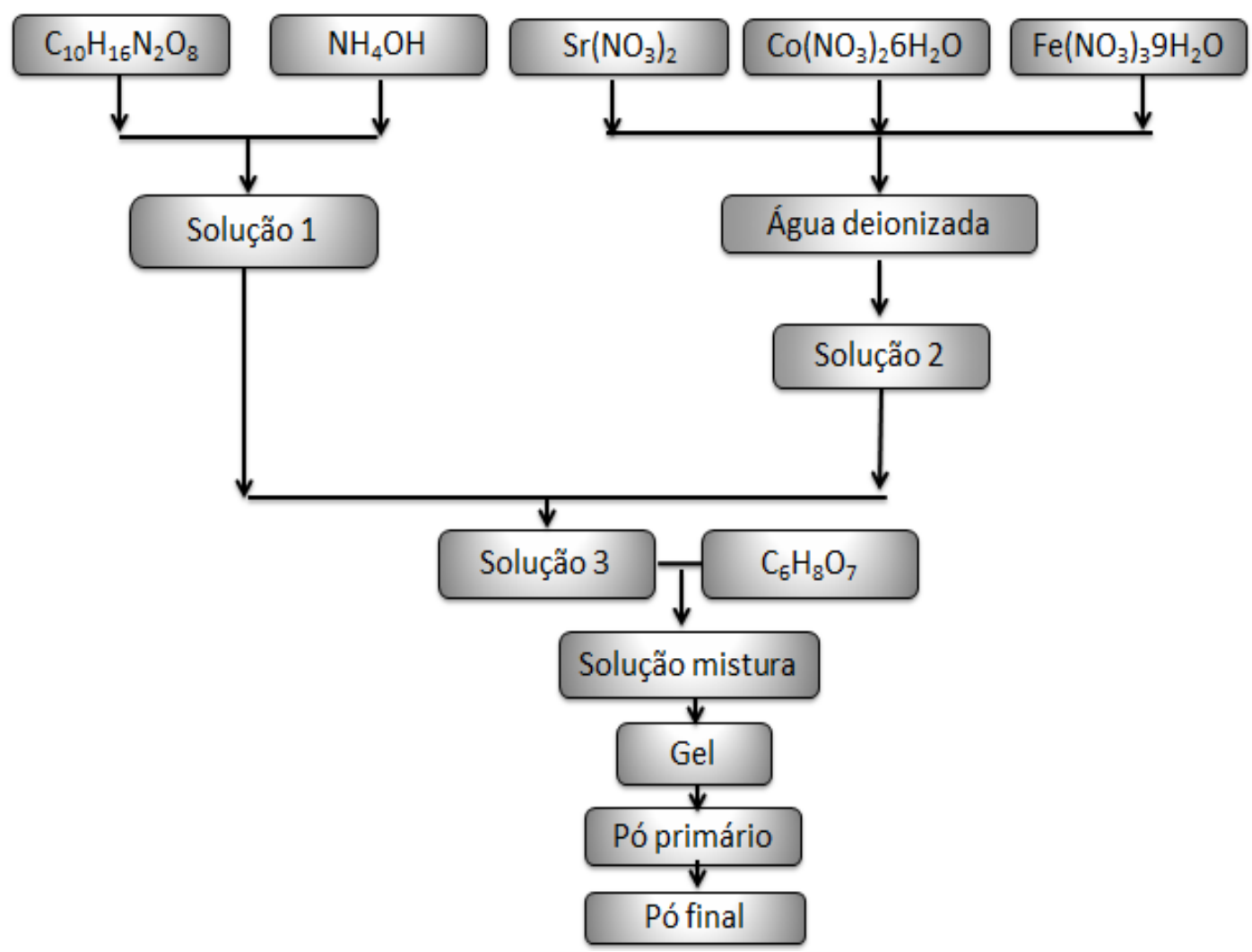

Figura 1 - Rota metodológica proposta para síntese dos materiais com base no método de complexação combinando EDTA - Citrato.

Cada um dos parâmetros que compõem a rota metodológica proposta, como: temperatura de cada etapa, tempo decorrente entre uma etapa e outra para a adição de reagente até a formação do gel foram inicialmente verificados. Após estudo realizado para montagem da rota metodológica da síntese dos materiais com base no método de complexação combinando EDTA - Citrato foram realizados experimentos modificando alguns parâmetros relevantes da síntese, como por exemplo: $\mathrm{pH}(6$ - 11) e condições de calcinação (taxa de aquecimento e tempo de permanência no patamar), no intuito de produzir um material monofásico, com o menor teor de impurezas (enxofre, carbono residual, entre outros) e menor tamanho médio de cristalito e para verificar a influência desses parâmetros no tamanho médio de cristalito do material. A razão molar de EDTA ácido, ácido cítrico e íons metálicos totais de 1:1,5:1 não foi verificada, pois trata-se de um parâmetro que não apresentou alteração nos 
diversos trabalhos encontrados na literatura, tais como os apresentados: Shao et al., (2001); Tong et al., (2002); Ikeguche et al., (2005); Dong et al., (2001); Rebeilleau et al., (2005); Lu et al., (2006). A Tabela 2 resume algumas das condições experimentais significativas adotadas para sínteses.

Tabela 2 - Condições experimentais de síntese para obtenção do $\mathrm{SrCo}_{0,8} \mathrm{Fe}_{0,2} \mathrm{O}_{3-\delta}$ a partir da rota metodológica proposta com base no método de complexação combinando EDTA-Citrato.

\begin{tabular}{c|c|c|c|c|c}
\hline \multirow{2}{*}{ EXPERIMENTO } & \multirow{2}{*}{$\begin{array}{c}\text { CODIFICAÇÃO } \\
\text { DAS AMOSTRAS }\end{array}$} & $\mathrm{pH}$ & $\begin{array}{c}\text { Pré-calcinação }\left({ }^{\circ} \mathrm{C}\right) / \\
\text { patamar }(\mathrm{h})\end{array}$ & $\begin{array}{c}\text { Taxa, } \\
\left({ }^{\circ} \mathrm{Cmin}^{-1}\right)\end{array}$ & $\begin{array}{c}\text { Calcinação }\left({ }^{\circ} \mathrm{C}\right) / \\
\text { Patamar }(\mathrm{h})\end{array}$ \\
\cline { 4 - 6 } & $\mathrm{TpH6}$ & 6 & $230 / 3$ & 2 & $950 / 5$ \\
\hline 1 & $\mathrm{TpH9}$ & 9 & $230 / 3$ & 2 & $950 / 5$ \\
\hline 2 & $\mathrm{TpH} 11$ & 11 & $230 / 3$ & 2 & $950 / 5$ \\
\hline 3 & $(4) \mathrm{a}$ & 11 & $230 / 3$ & 10 & $950 / 5$ \\
\hline 4 & $(5) \mathrm{a}$ & 11 & $230 / 5$ & 10 & $950 / 5$ \\
\hline 5 & & & & & \\
\hline
\end{tabular}

\subsubsection{Método de coprecipitação}

Um reator de mistura confeccionado em vidro borossilicato e montado em sistema de módulo composto por um motor/haste de agitação controlada por um inversor de frequência (CFW-08/WEQ) foi utilizado na síntese dos materiais a partir do método de coprecipitação em meio oxalato.

Devido ao controle de agitação no reator, foi inicialmente adicionado um volume de 200 $\mathrm{mL}$ de água deionizada ao reator, suficiente e necessário para agitação da solução, conforme dimensões pré-estabalecidas do reator de mistura utilizado para obtenção dos precursores da fase. Em seguida, a solução à base dos elementos que compõem a composição do material (Sr, $\mathrm{Co}$ e $\mathrm{Fe}$ ) juntamente com o agente precipitante e a trietilamina foram introduzidos por gotejamento simultâneo ao reator sob agitação continua à temperatura ambiente e mantida durante todo processo de síntese. A trietilamina foi adotada para controle do $\mathrm{pH} 11$ da mistura reacional. $\mathrm{O}$ valor do $\mathrm{pH}$ foi pré-definido em 11 para garantir a precipitação por completo de todos os constituintes da fase $\left(\mathrm{SrCO}_{0,8} \mathrm{Fe}_{0,2} \mathrm{O}_{3-\delta}\right)$.

A Figura 2 ilustra o Fluxograma da rota do processo de síntese adotado para obtenção dos precursores da fase com base no método via coprecipitação em meio oxalato e a Tabela 3 resume as condições de sínteses as quais foram realizados os experimentos.

Tabela 3- Condições experimentais adotadas para síntese dos precursores utilizando diferentes reagentes e agentes precipitantes com base no método via coprecipitação em meio oxalato.

\begin{tabular}{c|c|c}
\hline AMOSTRAS & AGENTE PRECIPITANTE & REAGENTES \\
\hline \multirow{2}{*}{1} & Trietilamina & $\mathrm{SrCO}$ \\
& $\left(\left(\mathrm{NH}_{4}\right)_{2} \mathrm{C}_{2} \mathrm{O}_{4} \mathrm{H}_{2} \mathrm{O} / \mathrm{C}_{2} \mathrm{H}_{2} \mathrm{O}_{4}\right)(1: 1)$ & $\mathrm{Co}\left(\mathrm{NO}_{3}\right)_{2} 6 \mathrm{H}_{2} \mathrm{O}$ \\
& Trietilamina & $\mathrm{Fe}\left(\mathrm{NO}_{3}\right)_{2} 6 \mathrm{H}_{2} \mathrm{O}$ \\
\hline \multirow{2}{*}{2} & $\left(\mathrm{NH}_{4}\right)_{2} \mathrm{C}_{2} \mathrm{O}_{4} \mathrm{H}_{2} \mathrm{O}$ & $\mathrm{SrCO}$ \\
& Trietilamina & $\mathrm{Co}\left(\mathrm{NO}_{3}\right)_{2} 6 \mathrm{H}_{2} \mathrm{O}$ \\
& $\mathrm{Fe}_{2}\left(\mathrm{C}_{2} \mathrm{O}_{4}\right)_{3} 6 \mathrm{H}_{2} \mathrm{O}$ \\
\hline \multirow{2}{*}{3} & $\mathrm{SrCO}_{3}$ \\
& $\left(\mathrm{NH}_{4}\right)_{2} \mathrm{C}_{2} \mathrm{O}_{4} \mathrm{H}_{2} \mathrm{O} / \mathrm{C}_{2} \mathrm{O}_{4} 2 \mathrm{H}_{2} \mathrm{O}(1: 1)$ & $\mathrm{Co}\left(\mathrm{NO}_{3}\right)_{2} 6 \mathrm{H}_{2} \mathrm{O}$ \\
\end{tabular}




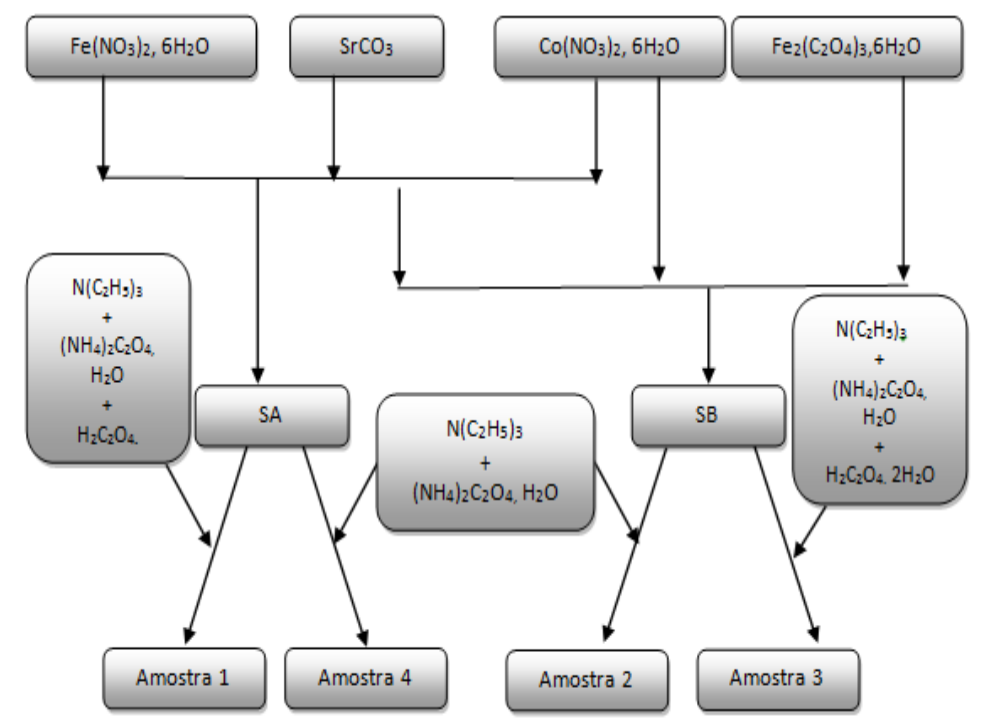

Figura 2 - Fluxograma o processo de síntese adotado para obtenção dos precursores da fase a partir do método via coprecipitação em meio oxalato

O precipitado obtido foi mantido em repouso durante um período de tempo mínimo necessário para a separação das fases constituintes (precipitado sólido e líquido resultante da síntese). Após esse período de repouso o precipitado foi separado e lavado várias vezes com água destilada até apresentar $\mathrm{pH}$ em torno de 7. Posteriormente,o precipitado foi seco em estufa a temperatura de aproximadamente $60{ }^{\circ} \mathrm{C}$ por um periodo de $24 \mathrm{~h}$ e finalmente, moído em almofariz. Os pós foram submetidos a tratamento térmico, nas seguintes condições: précalcinação a $230{ }^{\circ} \mathrm{C}$ por $180 \mathrm{~min}$ com taxa de aquecimento de $2{ }^{\circ} \mathrm{C} \mathrm{min}-1$ e calcinação a $950{ }^{\circ} \mathrm{C}$ por 300 min com taxa de aquecimento de $2{ }^{\circ} \mathrm{C} \mathrm{min}^{-1}$.

\subsection{Caracterizações dos pós}

Os pós cerâmicos obtidos pelos diferentes tipos de métodos de síntese foram caracterizados por Difração de Raios-X (DRX) pelo método do pó (Difratômetro de Raios-X Shimadzu DRX - 6000), utilizando radiação CuKa e comprimento de onda $\lambda$ igual a 1,5406 $\AA$, a fim de identificar as fases cristalinas. Os difratogramas foram obtidos na faixa $2 \theta$ de 20 a 80 graus em passos de 0,02 e tempo de passo de 1 segundo na temperatura ambiente de $25{ }^{\circ} \mathrm{C}$. A partir dos difratogramas foi realizado um refinamento pelo método Rietveld utilizando o software BDWS Tools 2.0 para determinação do tamanho médio de cristalito e outros parâmetros cristalográficos dos materiais obtidos a partir das alterações nos parâmetros de síntese. Os difratogramas de Raios-X foram comparados com a carta padrão $\mathrm{n}^{\circ}$ 79022- ICSD (Inorganic Crystal Structures Database) e a morfologia dos pós foram observadas a partir da microscopia eletrônica de varredura (MEV) no microscópio Philips XL 30 ESEM.

\section{RESULTADOS E DISCUSSÃO}

\subsection{Difrações de raios-X (DRX)}

As Figuras 3 e 4 apresentam os difratogramas da caracterização pelo método de complexação combinando EDTA - citrato, juntamente com o resultado do refinamento pelo 
método Rietveld referente à síntese do material cerâmico em condições distintas de $\mathrm{pH}$ da solução para obtenção do gel, a partir da rota proposta: (A) controle do pH em 6, (B) controle do $\mathrm{pH}$ em 9 e (C) controle do $\mathrm{pH}$ em 11 e estudo do tratamento térmico (patamar), conforme Tabela 3.2. A solução ao atingir o pH pré-definido, o mesmo foi controlado durante um período igual a $180 \mathrm{~min}$.
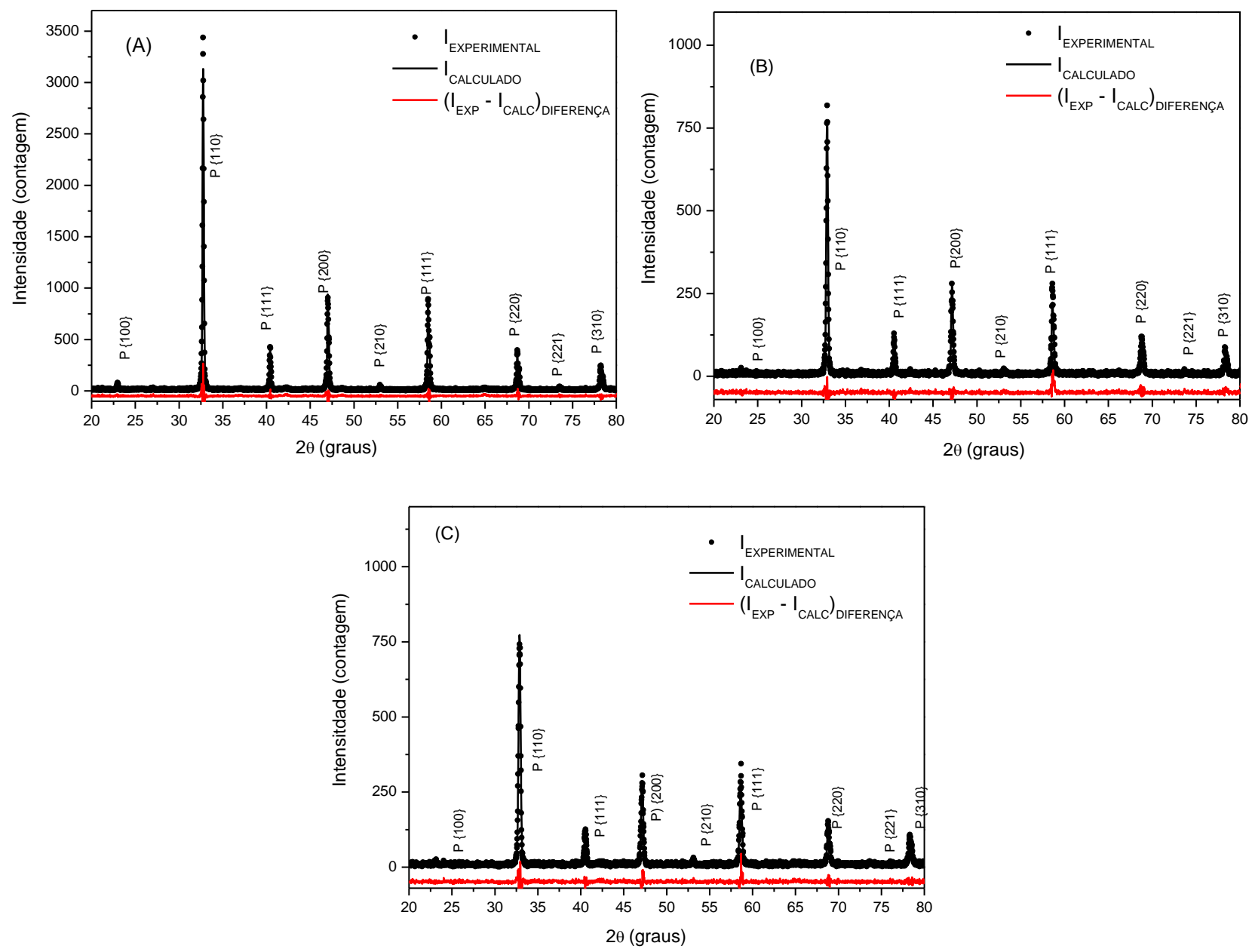

Figura 3 - Difratogramas do material $\mathrm{SrCo}_{0,8} \mathrm{Fe}_{0,2} \mathrm{O}_{3-\delta .}$ (A) pH 6, (B) pH 9 e (C) pH 11.
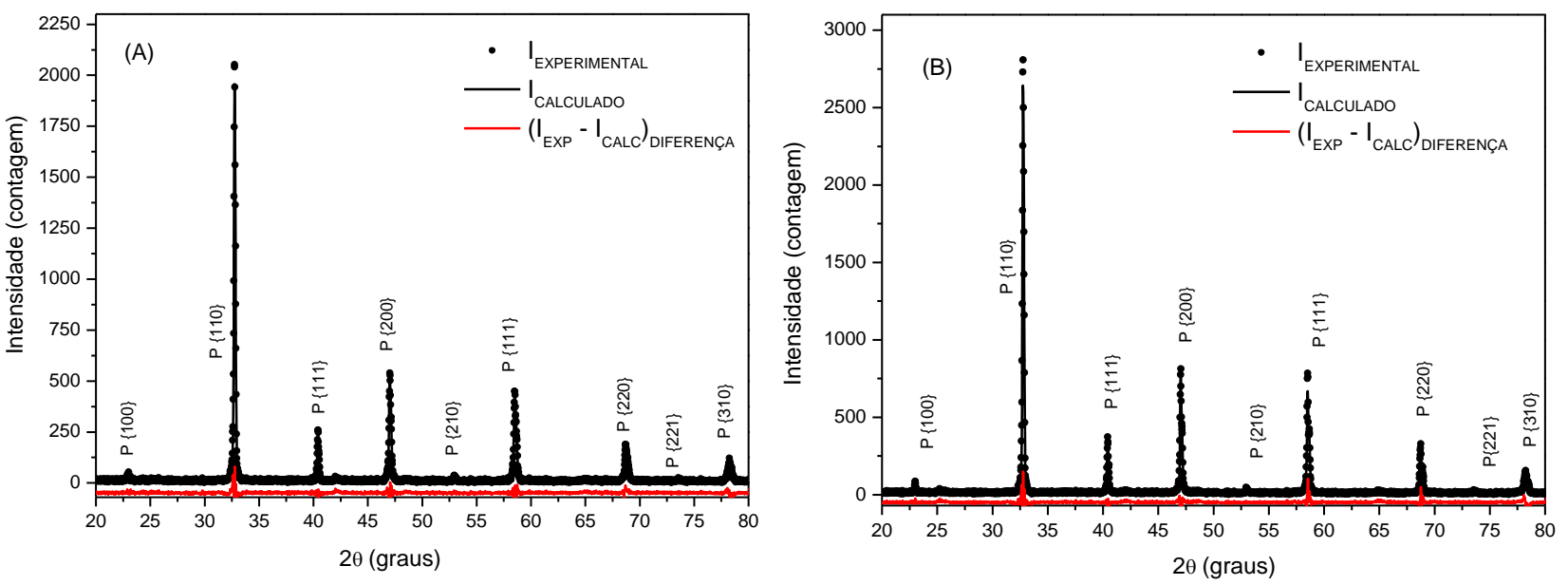

Figura 4 - Difratogramas do material $\mathrm{SrCO}_{0,8} \mathrm{Fe}_{0,2} \mathrm{O}_{3-\delta}$ referente ao estudo das condições de calcinação. Taxa de aquecimento: $10^{\circ} \mathrm{C} \mathrm{min}^{-1}$. Pré-calcinação: $230^{\circ} \mathrm{C}$ por: (A) $180 \mathrm{~min}$ e (B) $300 \mathrm{~min}$. 
Conforme os difratogramas apresentados nas Figuras 3 e 4 a fase $\mathrm{SrCo}_{0,8} \mathrm{Fe}_{0,2} \mathrm{O}_{3-\delta}$ foi formada independentemente do $\mathrm{pH}$ e do tratamento térmico adotado, conforme carta padrão $\mathrm{n}^{\circ}$ 79022- ICSD (Inorganic Crystal Structures Database).

A partir da análise dos resultados obtidos do refinamento Rietveld apresentados na Tabela 4 pode-se verificar a variação no tamanho médio de cristalito do material em função dos parâmetros modificados da síntese. Quando a síntese foi realizada em meio ácido ( $\mathrm{pH} \mathrm{6),} 0$ tamanho médio de cristalito é igual a $88,30 \mathrm{~nm}$ e esse valor diminui para 53,17 e 45,45 nm nos valores de $\mathrm{pH} 9$ e 11 , respectivamente.

Como os menores tamanhos de cristalitos ocorreram em $\mathrm{pH} 11$, outras sínteses foram realizadas variando a taxa de aquecimento $\left(2{ }^{\circ} \mathrm{C} \mathrm{min}^{-1}\right.$ e $\left.10{ }^{\circ} \mathrm{C} \mathrm{min}^{-1}\right)$ e o tempo de permanência no patamar de temperatura de calcinação (180 e $300 \mathrm{~min}$ ). Pode-se observar que o tamanho médio de cristalito variou com a taxa de aquecimento e também com o tempo de pré-calcinação, conforme resultados apresentados na Tabela 4. Aumentando o tempo de pré-calcinação de 180 min para 300 min o tamanho médio de cristalito também aumentou de 73,68 nm e 104,4 nm, respectivamente. A taxa de aquecimento apresentou comportamento semelhante ao tempo de pré-calcinação, como esperado, o tamanho médio de cristalito aumentou de 45,45 nm para 73,68 $\mathrm{nm}$ com o aumento na taxa de aquecimento de $2{ }^{\circ} \mathrm{C} \mathrm{min}{ }^{-1}$ para $10{ }^{\circ} \mathrm{C} \mathrm{min}^{-1}$. Provavelmente devido ao menor número de núcleos formados, tendo sido favorecida a fase de crescimento dos cristalitos com o aumento da taxa de aquecimento.

A análise dos parâmetros de rede, volume da célula unitária e densidade, apresentados na Tabela 4, revelam que os resultados encontrados através do refinamento pelo método Rietveld estão coerentes com aqueles citados na literatura (Harrison, 1995). O ajuste obtido pelo refinamento realizado pode ser confirmado pelo valor encontrado para o parâmetro residual (S), muito próximo da unidade. Portanto, os valores obtidos através do refinamento pelo método Rietveld são considerados confiáveis.

Tabela 4- Parâmetros de rede, volume de célula, densidade e tamanho médio de cristalito das amostras obtidas a partir do refinamento Rietveld.

\begin{tabular}{|c|c|c|c|c|c|}
\hline EXPERIMENTO & (1) & (5) & (6) & $(13)$ & $(15)$ \\
\hline Parâmetros de rede $(a=b=c)$ & 3,8590 & 3,8618 & 3,8598 & 3,8575 & 3,8583 \\
\hline Volume de célula, $\AA$ & 57,470 & 57,595 & 57,506 & 57,401 & 57,437 \\
\hline Densidade, $\mathrm{Gr} \mathrm{Cm}^{-3}$ & 5,505 & 5,493 & 5,502 & 5,512 & 5,508 \\
\hline Tamanho médio de cristalito, $\mathrm{nm}$ & 88,30 & 53,17 & 45,45 & 73,68 & 104,4 \\
\hline Parâmetro Residual, S & 1,30 & 1,24 & 1,16 & 1,27 & 1,30 \\
\hline
\end{tabular}

Conforme os resultados de difratometria e refinamento Rietveld apresentados, a fase $\left(\mathrm{SrCO}_{0,8} \mathrm{Fe}_{0,2} \mathrm{O}_{3-\delta}\right)$ foi obtida em todas as condições de síntese apresentadas. Todavia, observa-se que as propriedades cristalográficas, tamanho médio de cristalito do material são afetados de acordo com as alterações realizadas no pH da solução para obtenção do gel e tratamento térmico para obtenção do pó. Sendo o pH 11 a condição de síntese que mais favoreceu para formação de pós com menor tamanho médio de cristalito $(45,45 \mathrm{~nm})$. Do ponto de vista aplicativo do material $\mathrm{SrCO}_{0,8} \mathrm{Fe}_{0,2} \mathrm{O}_{3-\delta}$ (SCFO), por exemplo, em processos industriais que requerem um fornecimento continuo de oxigênio puro, tais como combustível oxidante em célula combustível e catalisadores para reações de combustão de hidrocarbonetos, a condição de síntese que favorece a formação 
do material com menor tamanho médio de cristalito, dentre os aqui apresentados foi: $\mathrm{pH} 11$, pré-calcinação a $230{ }^{\circ} \mathrm{C}$ por $180 \mathrm{~min}$ com taxa de aquecimento de $2{ }^{\circ} \mathrm{C} \mathrm{min}{ }^{-1}$ e calcinação a $950{ }^{\circ} \mathrm{C}$ por $300 \mathrm{~min}$ com taxa de aquecimento de $2{ }^{\circ} \mathrm{C} \mathrm{min}^{-1}$. Todavia, faz-se ainda necessário a utilização de um processo de moagem para uma diminuição e homogeneização do tamanho de partícula.

Os difratogramas de Raios-X do material obtido pelo método de coprecipitação em meio oxalato, juntamente como o refinamento pelo método Rietveld referente à síntese do material cerâmico nas quatro condições distintas de síntese são apresentados na Figura 5.
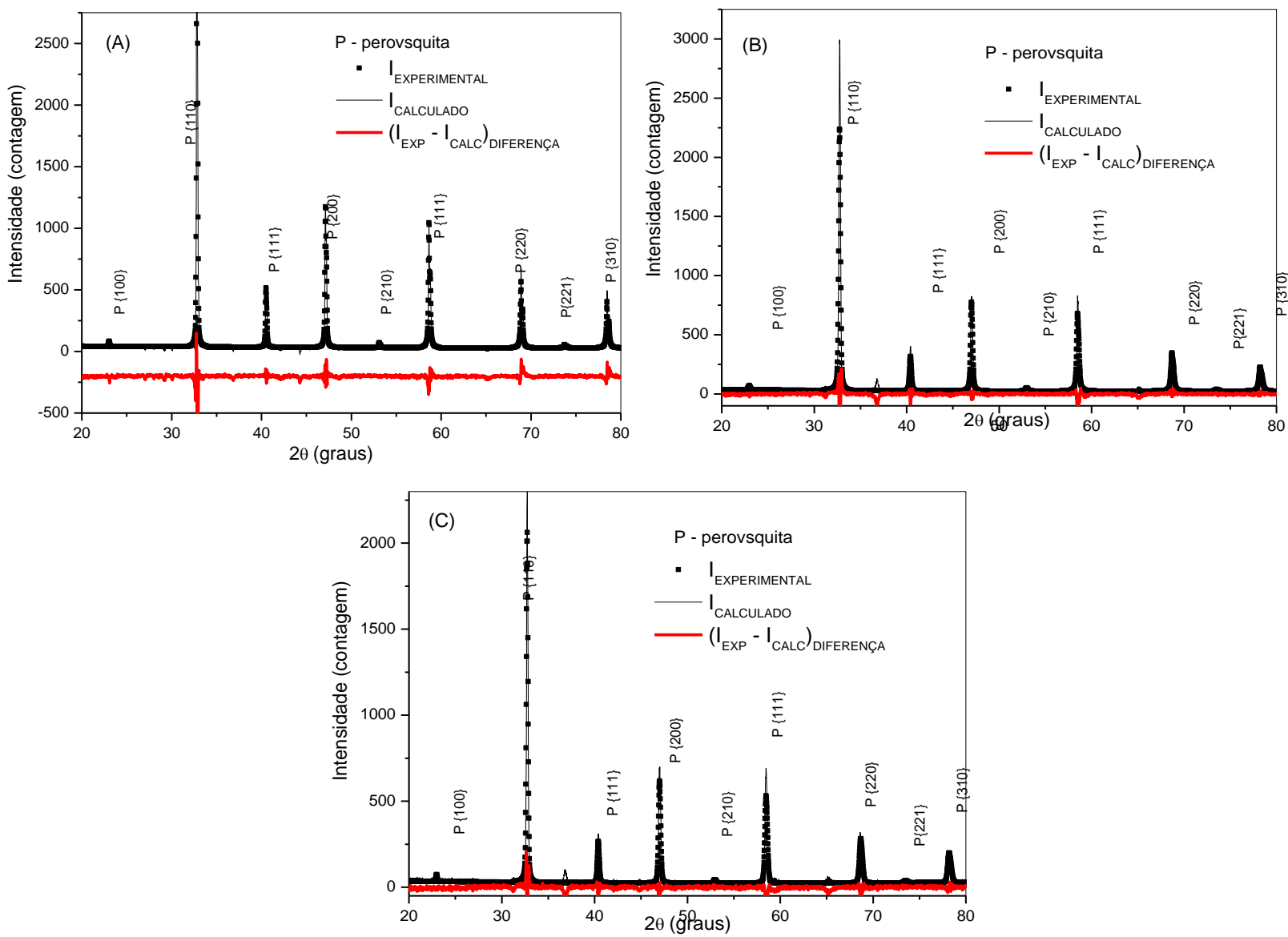

Figura 5 - Difratogramas referente à síntese do material cerâmico em 4 condições distintas de síntese (Tabela 3): (A) Amostra 1, (B) Amostra 2 e (C) Amostra 3.

De acordo com os difratogramas de raios - $X$ e refinamento Rietveld apresentados, a fase $\left(\mathrm{SrCO}_{0,8} \mathrm{Fe}_{0,2} \mathrm{O}_{3-\delta}\right)$ foi obtida em todas as condições de síntese, mas com uma alteração na cristalinidade do material obtido quando substituímos um dos reagentes adotados na síntese; nitrato de ferro III nonohidratado utilizado na síntese 1 pelo oxalato de ferro III hexahidratado na amostra 3. Todavia, no caso da amostra 4, o refinamento obtido a partir do método Rietveld apresentou um parâmetro residual igual a 3,57 (diferença entre os pontos experimentais obtido a partir da síntese e o calculado a partir da carta padrão e o refinamento pelo método Rietveld) significativo, superior ao das demais amostras (1-3), sendo assim necessário uma análise mais elaborada desta condição de síntese na obtenção da fase. Dessa forma, as demais caracterizações apresentadas são correspondentes apenas as amostras (1-3).

A utilização do nitrato de ferro III nonohidratado na condição de amostra 1 apresentou um material com maior cristalinidade quando comparado com o resultado obtido na amostra 3 , 
conforme Figura $5(A)$ e $(C)$, respectivamente. Tal comportamento possivelmente seja atribuído a diferença de solubilidade existente entre os nitratos e os oxalatos; uma vez que os nitratos são completamente solúveis em água e os oxalatos não. Existe, uma situação mais favorável para o crescimento do tamanho médio dos cristalitos, sendo os nitratos mais reativos. Havendo dessa forma, um tempo maior para o grão se desenvolver e consequentemente a geração de grãos com maior tamanho médio de cristalito. Enquanto, com o uso dos oxalatos, como são parcialmente solúveis em água, é gerado de certa forma uma resistência para reação e, por conseguinte, um maior tempo de reação se fará necessário não tendo condições propícias para o grão se desenvolver e, portanto, menores tamanhos dos cristalitos serão formados.

O uso de nitrato de metais propicia melhores condições de síntese, pois ele tem uma cinética de decomposição mais favorável comparado aos carbonatos correspondentes. Isto leva a uma redução da temperatura de calcinação.

Segundo os difratogramas apresentados na Figura 5 (B) e (C), pode-se observar que o agente precipitante adotado na síntese praticamente não influenciou na formação da fase $\mathrm{SrCO}_{0,8} \mathrm{Fe}_{0,2} \mathrm{O}_{3-\delta}$. Nota-se ainda a partir dos resultados apresentados nos difratogramas uma pequena variação na distância interplanar entre os dados experimentais e a carta padrão (Harrison, 1995), possivelmente atribuído a condições experimentais distintas.

A Tabela 5 apresenta os parâmetros de rede, densidade e tamanho médio de cristalito das amostras obtidas a partir das condições experimentais de síntese adotadas (1-3) obtidos a partir dos difratogramas e do refinamento pelo método Rietveld.

Tabela 5 - Parâmetros de rede, densidade e tamanho médio de cristalito dos materiais obtidos a partir do refinamento Rietveld.

\begin{tabular}{c|c|c|c|c}
\hline AMOSTRA & $\begin{array}{c}\text { PARÂMETROS DE REDE } \\
(\mathrm{a}=\mathrm{b}=\mathrm{c}), \\
\AA\end{array}$ & $\begin{array}{c}\text { DENSIDADE, } \\
\mathrm{g} \cdot \mathrm{cm}^{-3}\end{array}$ & $\begin{array}{c}\text { TAMANHO } \\
\text { MÉDIO DE } \\
\text { CRISTALITO, } \mathrm{nm}\end{array}$ & $\begin{array}{c}\text { PARÂMETRO } \\
\text { RESIDUAL, } \\
\text { S }\end{array}$ \\
\hline 1 & 3,84 & 5,55 & 77,22 & 1,60 \\
\hline 2 & 3,86 & 5,50 & 42,95 & 1,68 \\
\hline 3 & 3,86 & 5,49 & 42,23 & 1,50 \\
\hline
\end{tabular}

A partir dos resultados apresentados na Tabela 5 pode-se verificar a variação no tamanho médio de cristalito em função dos parâmetros de síntese modificados. Quando a síntese foi realizada com o nitrato de ferro III nonohidratado (síntese 1) o tamanho médio de cristalito foi aproximadamente $77,22 \mathrm{~nm}$ quase duas vezes maior que quando se utilizou o oxalato de ferro III hexahidratado na síntese $3(42,23 \mathrm{~nm})$ e a mistura equimolar de $\left(\mathrm{NH}_{4}\right)_{2} \mathrm{C}_{2} \mathrm{O}_{4} \mathrm{H}_{2} \mathrm{O}$ e $\mathrm{C}_{6} \mathrm{H}_{8} \mathrm{O}_{7}$ como agente precipitante e na síntese $2(42,95 \mathrm{~nm})$ utilizando apenas o $\left(\mathrm{NH}_{4}\right)_{2} \mathrm{C}_{2} \mathrm{O}_{4} \mathrm{H}_{2} \mathrm{O}$ como agente precipitante. Este resultado está de acordo com os difratogramas apresentados na Figura 5 (A) e (C), ou seja, a condição de síntese 1 que apresentou maior cristalinidade também se obteve maior tamanho médio de cristalito. A diferença de aproximadamente $80 \%$ do tamanho médio de cristalito entre as sínteses 1 e 3 é devido provavelmente a diferença entre as solubilidades do nitrato e oxalato de ferro (III) que influenciam na nucleação dos grãos durante o processo de síntese.

Já no caso onde houve mudança no agente precipitante adotado na síntese, o tamanho médio de cristalito praticamente não variou (42,95 nm e 42,23 nm), conforme síntese 2 e 3, respectivamente, quando comparados com os reagentes de mesma natureza. 
A análise dos parâmetros de rede, célula unitária e densidade, apresentados na Tabela 5, revelam que os resultados encontrados no refinamento estão coerentes com aqueles citados em Harrison, (1995). O ajuste obtido pelos refinamentos realizados pode ser confirmado pelo valor encontrado para o parâmetro residual (S) muito próximo da unidade, com exceção da amostra 4. Portanto, os valores obtidos pelo refinamento Rietveld são considerados confiáveis. No caso da amostra 4, o parâmetro residual é superior a 3, apresentado pelo refinamento a partir do método Rietveld não demonstra confiabilidade do resultado obtido.

Conforme os resultados de difratometria e refinamento Rietveld apresentados, a fase $\left(\mathrm{SrCO}_{0,8} \mathrm{Fe}_{0,2} \mathrm{O}_{3-\delta}\right)$ foi obtida nas em todas as condições de síntese adotadas. Todavia, observa-se que a cristalinidade e o tamanho médio de cristalito do material são afetados de acordo com as alterações realizadas, seja no agente precipitante ou a natureza do reagente adotado.

A síntese com base no método via coprecipitação em meio oxalato nos permitiu obter grãos com uma faixa média de tamanho médio de cristalito entre aproximadamente $42 \mathrm{~nm}$ e 77 $\mathrm{nm}$, conforme as condições de síntese empregada. Tais resultados comparados com o obtido a partir do método de complexação combinando EDTA - Citrato, cujo foi adotado as mesmas condições do tratamento térmico, foi observado que na síntese realizada por este último método foi obtida grãos com tamanho médio de cristalito na faixa de $45 \mathrm{~nm}$, mesma ordem de grandeza que o obtido pelo método via co-precipitação em meio oxalato. Todavia, Rui et al., (2010) sintetizaram a perovsquita $\mathrm{SrCO}_{0,8} \mathrm{Fe}_{0,2} \mathrm{O}_{3-\delta}$ a partir do método dos citratos, com temperatura de calcinação igual a $900{ }^{\circ} \mathrm{C}$ e observaram aglomerados de partículas com tamanho entre 8 e $53 \mu \mathrm{m}$.

\subsection{Microscopia Eletrônica de Varredura (MEV)}

A Figura 6 apresenta os resultados da análise de microscopia eletrônica de varredura (MEV) da amostra ternária $\mathrm{SrCo}_{0,8} \mathrm{Fe}_{0,2} \mathrm{O}_{3-\delta}$ obtida pelo método de complexação combinando EDTA - Citrato a partir da condição de síntese (1), conforme Tabela 2. A micrografia da amostra passou por uma ampliação de 5000 x (A) e 15000 x (B).
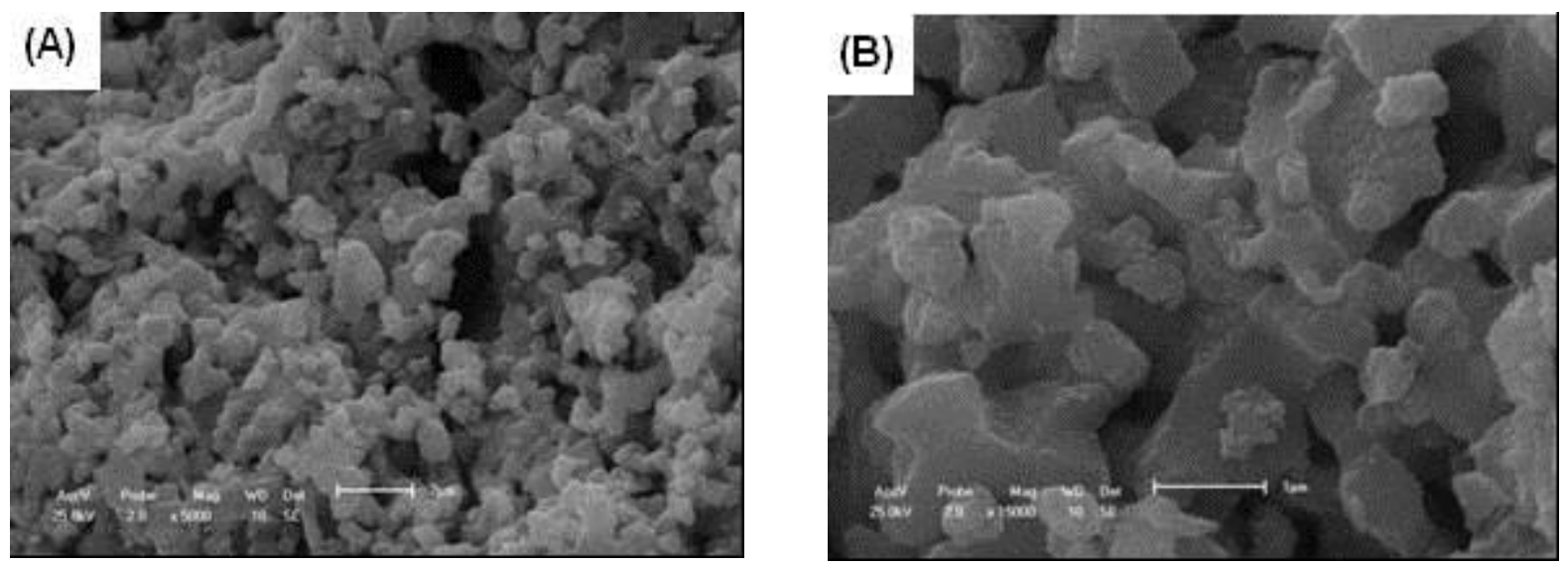

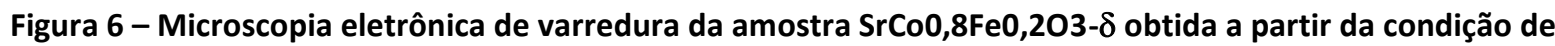
síntese (1) (pH 6) com base no método de complexação combinando EDTA - Citrato. Ampliação (A) 5000x e (B) 15000x.

A morfologia do pó cerâmico do tipo perovsquita $\mathrm{SrCo}_{0,8} \mathrm{Fe}_{0,2} \mathrm{O}_{3-\delta}$ foi verificada a partir da microestrutura dos pós cerâmicos, conforme ampliações da amostra ilustrada na Figura 6. 
Observam-se aglomerados de partículas de tamanho não uniforme, assim como ocorreu nas outras condições de síntese. Resultados semelhantes aos apresentados por Rui et al., (2010).

A Figura 7 apresenta os resultados da análise de microscopia eletrônica de varredura (MEV) do material $\left(\mathrm{SrCo}_{0,8} \mathrm{Fe}_{0,2} \mathrm{O}_{3-\delta}\right)$ obtidos a partir do método de coprecipitação em meio oxalato nas condições de síntese (1-3), conforme Tabela 3, obtidas a partir das condições: $\mathrm{pH}$ 11, pré-calcinação a $230{ }^{\circ} \mathrm{C}$ por $180 \mathrm{~min}$ com taxa de aquecimento de $2{ }^{\circ} \mathrm{C} \mathrm{min}{ }^{-1}$ e calcinação a $950{ }^{\circ} \mathrm{C}$ por $300 \mathrm{~min}$ com taxa de aquecimento de $2{ }^{\circ} \mathrm{C} \mathrm{min}{ }^{-1}$. A micrografia do material passou por uma ampliação igual a $5000 \mathrm{x}$.
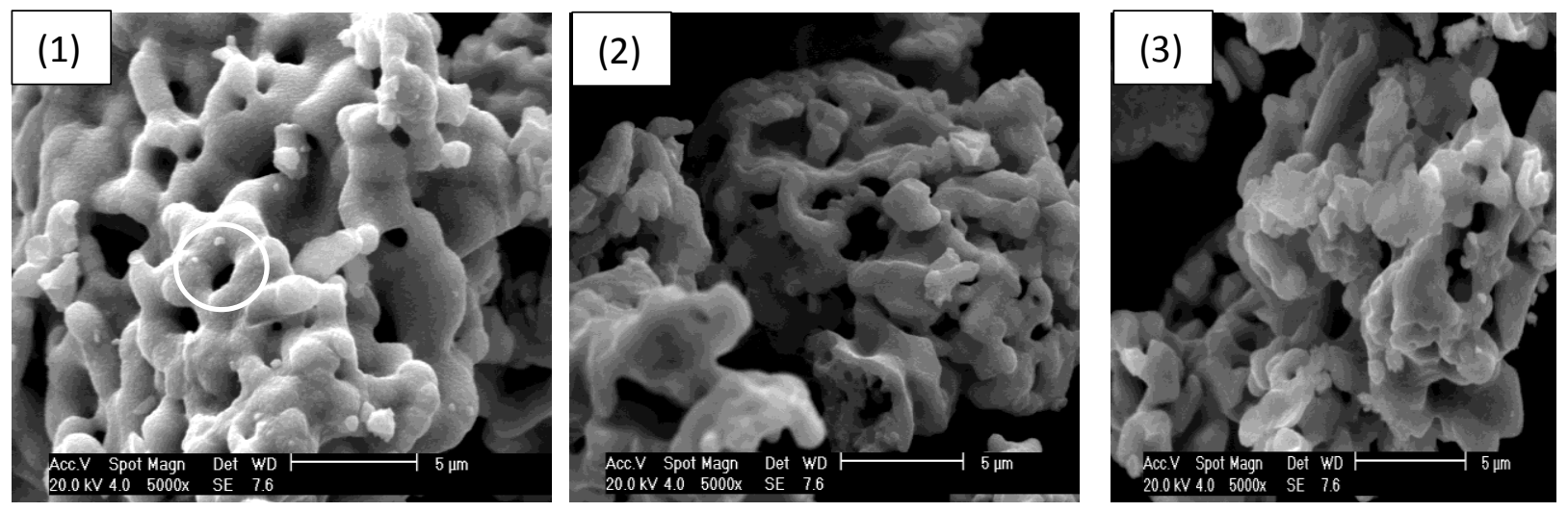

Figura 7 - Microscopia eletrônica de varredura do material (SrCo0,8Fe0,203- $\delta$ ) referente as amostras (1-3). Ampliação: 5000 x.

A partir da Figura 7, pode-se observar partículas de tamanho não uniforme, sendo a amostra composta de aglomerados de partículas, notando também a presença de poros e formação de pescoços, conforme destacado na Figura 7 (1). Resultados corroborantes com os apresentados por Rui et al., (2010).

Independentemente do método de síntese (método de complexação combinando EDTA Citrato apresentado primeiramente e método de coprecipitação via oxalato abordado posteriormente), a morfologia dos pós apresentam formatos semelhantes com aglomeradas de partículas não homogêneas.

\section{CONCLUSÕES}

A partir do difratograma de raios $-X$ e refinamento pelo método Rieltveld verificou-se que o tamanho médio de cristalito do material variou conforme as distintas condições de síntese adotada, dos diferentes métodos utilizados. Foi permitido pelo método de complexação combinando EDTA - Citrato obter pós monofásicos de tamanho médio de cristalito de 45,45 nm, e na síntese com base no método de coprecipitação em meio oxalato foi observado que a natureza do reagente adotado na síntese teve maior influência no tamanho médio de cristalito $(77,22 \mathrm{~nm}$ e $42,23 \mathrm{~nm})$ que no agente precipitante adotado $(42,23 \mathrm{~nm}$ e $42,95 \mathrm{~nm})$. Entretanto, a morfologia do pó também não apresentou variação significativa com a mudança no método adotado para síntese do material, apresentando partículas e aglomerados de partículas não uniformes. Contudo, o método via coprecipitação em meio oxalato apresentou a vantagem de obter excelentes rendimentos do material sintetizado (90 a $95 \%$ ), enquanto que o obtido pelo método de complexação combinando EDTA - Citrato da rota de síntese estudada neste trabalho 
foi entre 65 e $75 \%$, mantendo o tamanho médio de cristalito na mesma ordem de grandeza. Outras vantagens observadas a partir do método de coprecipitação via oxalato é a obtenção de precursores sólidos em menor período de síntese, em menor temperatura $\left(26^{\circ} \mathrm{C}\right)$, e com etapas independentes (síntese dos precursores, lavagens, secagem e calcinação.

\section{REFERÊNCIAS BIBLIOGRÁFICAS}

1. DONG H., SHAO Z., XIONG G., TONG J., SHENG S., YANG W., Investigation on POM reaction in a new perovskite membrane reactor, Catalysis Today, 67, 3-13, 2001.

2. FAN C. G., DENG Z. Q., ZUO Y. B. LIU W., CHEN C. S., Preparation and characterization of $\mathrm{SrCo}_{0.8} \mathrm{Fe}_{0.2} \mathrm{O}_{3-\delta}$ oxygen permeable composite membrane, Solid State Ionics, 166, 339-342, 2004.

3. FAN C., LIU W., DENG Z., ZUO Y., CHEN C., BAE D., Mechanical stability and transport properties of the Sn-promoted $\mathrm{SrCO}_{0.8} \mathrm{Fe}_{0.2} \mathrm{O}_{3-\delta}$ ceramic membrane, Journal of Membrane Science, 290, 73-77, 2007.

4. HARRISON W.T.A., LEE T.H., YANG Y.L., SCARFE D.P., LIU L.M., JACOBSON A.J., A neutron diffraction study of two strontium cobalt iron oxides, Materials Research Bulletin, 30, 621 630, 1995.

5. IKEGUCHE M., MIMURA T., SEKINE Y., KIKUCHI E., MATASUKATA M., Reaction and oxygen permeation studies in $\mathrm{Sm}_{0.4} \mathrm{Ba}_{0.6} \mathrm{Fe}_{0.8} \mathrm{Co}_{0.2} \mathrm{O}_{3-\delta}$ membrane reactor for partial oxidation of methane to syngas, Applied Catalysis A: General 290, 212-220, 2005.

6. IKEGUCHI M.; YOSHINO Y.; KANIE K.; NOMURA M.; KIKUCHI E.; MATSUKATA M.; Effects of preparation method on oxygen permeation properties of $\mathrm{SrFeCo}_{0.5} \mathrm{O}_{\mathbf{x}}$ membrane, Separação e Purificação Tecnologia, 32, 313-318, 2003.

7. LEE T. H., YANG Y. L., JACOBSON A. J., ABELES B., MILNER S., Oxygen permeation in $\mathrm{SrCO}_{0.8} \mathrm{Fe}_{0.2} \mathrm{O}_{3-\delta}$ membranes with porous electrodes, Solid State lonics, 100, 87-94, 1997.

8. LIMA S. M. e ASSAF J. M., Síntese e caracterização de perovskitas $\mathrm{LaNi}_{(1-\mathrm{x})} \mathrm{Co}_{\mathrm{x}} \mathrm{O}_{3}$ como precursores de catalisadores para a conversão do metano a gás de síntese pela reforma com $\mathrm{CO}_{2}$, Química Nova, 30, 298-303, 2007.

9. LIU L. M., LEE T. H., YANG Y. L., AND JACOBSON A. J., A themogravimetric study of the phase diagram of strontium cobalt iron oxide, $\mathrm{SrCO}_{0.8} \mathrm{Fe}_{0.2} \mathrm{O}_{3-\delta}$, Materials Research Bulletin, 31, 29$35,1996$.

10. LU H., CONG Y., YANG W.S., Oxygen permeability and stability of $\mathrm{Ba}_{0.5} \mathrm{Sr}_{0.5} \mathrm{Co}_{0.8} \mathrm{Fe}_{0.2} \mathrm{O}_{3-\delta}$ as an oxygen-permeable membrane at high pressures, Solid State lonics, 177, 595 - 600 ,2006.

11. MACLEOD, C. S.; HARVEY, A. P.; LEE, A. F.; WILSON, K. Evaluation of the activity and stability of alkali-doped metal oxide catalysts for application to an intensified method of biodiesel production. Chemical Engineering Journal, 135, 1-2, 63-70, 2008.

12. MADHUVILAKKU R. E PIRAMAN S., Biodiesel synthesis by $\mathrm{TiO}_{2}-\mathrm{ZnO}$ mixed oxide nanocatalyst catalyzed palm oil transesterification process/Bioresource Technology 150, 55-59, 2013.

13. MCINTOSH S., VENTE J. F., HAIJE W. G., DAVE B. H.A., Bouwmeester H. J. M., Structure and oxygen stoichiometry of $\mathrm{SrCo}_{0.8} \mathrm{Fe}_{0.2} \mathrm{O}_{3-\delta}$ and $\mathrm{Ba}_{0.5} \mathrm{Sr}_{0.5} \mathrm{Co}_{0.8} \mathrm{Fe}_{0.2} \mathrm{O}_{3-\delta}$, Solid State Ionics, 177, 1737-1742, 2006.

14. MINESHIGE A. IZUTSU, NAKAMURA M., NIGALI K., ABE J., KOBUNE M., FUJJI S., YAZAWA T., 
Introduction of A-site deficiency into $\mathrm{La}_{0.6} \mathrm{Sr}_{0.4} \mathrm{Co}_{0.2} \mathrm{Fe}_{0.8} \mathrm{O}_{3-\delta}$, Solid State Ionics,176, 11451149, 2005.

15. NAKATANI, N.; TAKAMORI, H.; TAKEDA, K.; SAKUGAWA, H.; Transesterification of soybean oil using combusted oyster shell waste as a catalyst, Bioresource Technology, 100, 3, 1510-1513, 2009.

16. QIU L., ICHIKAWA T., HIRANO A., IMANISHI N., TAKEDA Y., $\mathrm{Ln}_{1-x} \mathrm{Sr}_{x} \mathrm{CO}_{1-y} \mathrm{Fe}_{y} \mathrm{O}_{3-\delta}$ (Ln=Pr, Nd, Gd; $x=0.2,0.3)$ for the Electrodes of Solid Oxide Fuel Cells, Solid State Ionics, 158, 55-65, 2003.

17. QIU L., LEE T.H., LIU L. M., YANG Y.L., JACOBSON A.J., Oxygen permeation studies of $\mathrm{SrCO}_{0.8} \mathrm{Fe}_{0.2} \mathrm{O}_{3-\delta}$, Solid State lonics, 76, 321-329, 1995.

18. RANGEL M. C.; Membranas inorgânicas e reatores catalíticos, Química nova, 20, 486-492, 1997.

19. REBEILLEAU-DASSONNEVILLE M., ROSINI S., VAN VEEN A.C., FARRUSSENG D., MIRODATOS C., Oxidative activation of ethane on catalytic modified dense ionic oxygen conducting membranes, Catalysis Today, 104, 131-137,2005.

20. RUI Z., DING J., LI Y., LIN Y.S., $\mathrm{SrCO}_{0.8} \mathrm{Fe}_{0.2} \mathrm{O}_{-\delta}$ sorbent for high-temperature production of oxygen-enriched carbon dioxide stream, Fuel, 89, 1429-1434, 2010.

21. SANTOS, A. G; MADJID, A.; PATOUT, L.; SOUSA, C. P. de, $\mathrm{LaNi}_{0.3} \mathrm{Co}_{0.7} \mathrm{O}_{3-\delta}$ and $\mathrm{SrFe}_{0.2} \mathrm{CO}_{0.8} \mathrm{O}_{3-\delta}$ Ceramic Materials: Structural and Catalytic Reactivity under CO Stream, Catalysts, 4, p. 7788, 2014.

22. SHAO Z., DONG H., XIONG G., CONG Y. AND YANG W., Performance of a mixed-conducting ceramic membrane reactor with high oxygen permeability for methane conversion, Journal of Membrane Science, 183, 2-1, 181-192, 2001.

23. SOARES A. B., SILVA P. R. N., FREITAS J. C. C., ALMEIDA C. M., Estudo da oxidação total do etanol usando óxidos tipo perovskita ( $B=\mathrm{Mn}, \mathrm{Ni}, \mathrm{Fe}$ ), Química Nova, 30, 1061-1066, 2007.

24. STATHOPOULOS V.N., BELESSI V.C., BAKAS T.V., NEOPHYTIDES S.G., COSTA C.N., POMONIS P.J., EFSTATHIOU A.M., Comparative Study of La-Sr-Fe-O Perovskite-type Oxides Prepared by Ceramic and Surfactant Methods over the $\mathrm{CH}_{4}$ and $\mathrm{H}_{2}$ Lean-deNOx, Applied Catalysis B: Environmental, 93, 1-11, 2009.

25. SU MENGXING, ANG RU Y, LI MIN, Biodiesel production from hempseed oil using alkaline earth metal oxides supporting copper oxide as bi-functional catalysts for transesterification and selective hydrogenation, Fuel 103,398-407, 2013.

26. TERAOKA Y., HUA-MIN ZHANG, FURUKAWA S.; YAMAZOI N., Oxygen permeation through perovskite-type oxides, 14, $1743-1746,1985$.

27. TERAOKA Y., NOBUNAGA T., YAMOZOE N., Effect of cation substitution on the oxygen semipermeability of perovskite-type oxides, Chemical Letters, 503-506,1988.

28. TONG J., YANG W., CAI R., ZHU B., LIN L., Novel and ideal zirconium-based dense membrane reactors for partial oxidation of methane to syngas, Catalysis Letters, 78, 129-137, 2002.

29. YAN, S.; SALLEY, S. O.; SIMON NG, K. Y.; Simultaneous transesterification and esterification of unrefined or waste oils over $\mathrm{ZnO}-\mathrm{La}_{2} \mathrm{O}_{3}$ catalysts, Applied Catalysis A: General, 353, 203-212, 2009.

30. ZHANG K. YANG Y. L., PONNUSAMY D. JACOBSON A. J. SALAMA K., Effect of microstructure on oxygen permeation in $\mathrm{SrCO}_{0.8} \mathrm{Fe}_{0.2} \mathrm{O}_{3-\delta}$, journal of materials science, 34, 1367-1372, 1999. 